Check for updates

Cite this: Mater. Adv., 2022, 3,3571

Received 2nd August 2021, Accepted 23rd February 2022

DOI: 10.1039/d1ma00678a

rsc.li/materials-advances

\section{Oxygen vacancies in oxidized and reduced vertically aligned $\alpha-\mathrm{MoO}_{3}$ nanoblades $\dagger$}

\author{
Sohaila Z. Noby, ${ }^{\star a b}$ Azhar Fakharuddin, ${ }^{a}$ Stefan Schupp, ${ }^{a}$ Muhammad Sultan, ${ }^{c}$ \\ Marina Krumova, ${ }^{d}$ Malte Drescher, (D) ${ }^{d}$ Mykhailo Azarkh, (D) ${ }^{d}$ Klaus Boldt (D) ${ }^{e}$ and \\ Lukas Schmidt-Mende (D)*a
}

\begin{abstract}
Functionalized materials are highly desired for technological advancements spanning physics, chemistry, materials science, and biology due to their unique electronic properties. One such example is molybdenum trioxide $\left(\mathrm{MoO}_{3}\right)$, a metal oxide with multiple oxidation states. Manipulating these oxidation states can alter the electronic properties, for instance, defects and electrical conductivity, by several orders of magnitude. In this work, oxygen vacancy-mediated intrinsic defects in vertically aligned $\alpha-\mathrm{MoO}_{3}$ crystals are systematically tuned via thermal treatment under different reducing and oxidizing atmospheres. The positions and the concentration of the oxygen vacancies and restitution of the oxygen ions have been experimentally demonstrated via a range of techniques including electron paramagnetic resonance, X-ray diffraction, and high-resolution electron microscopy. The calculated concentration of the oxygen vacancies in the $\alpha-\mathrm{MoO}_{3-x}$ via EPR measurements is in the range of $x=0.004-0.049$. The mechanism of the formation of oxygen vacancies in the $\alpha-\mathrm{MoO}_{3-x}$ crystal is understood via color center formation and polaron migration models. These oxygen vacancies show no influence on the optical band gap. However, they significantly impact the electrical conductivity on the order of $10^{2} \mathrm{Sm}^{-1}$ by altering the $\mathrm{MoO}_{3}$ properties from semi-insulating to conducting.
\end{abstract}

\section{Introduction}

Transition-metal oxides are widely employed in organic photovoltaics and organic light emitting diodes because of their exceptional charge transport/extraction and injection properties between the metal oxide and absorber/emitter layer, respectively. ${ }^{1}$ This is because the electronic properties of metal oxides e.g., conductivity and energetic alignment with the absorber/emitter layer, can be easily tuned. As an example, $\mathrm{MoO}_{3}$ can simultaneously overcome two key issues of polyethylene dioxythiophene:polystyrene sulfonate (PEDOT:PSS), one of the most applied organic hole transport materials in solution processed solar cells: low chemical stability and low work function (5.0-5.1 eV). ${ }^{2} \mathrm{MoO}_{3}$ offers a higher and tunable work

\footnotetext{
${ }^{a}$ Department of Physics, University of Konstanz, 78467 Konstanz, Germany. E-mail: lukas.schmidt-mende@uni-konstanz.de

${ }^{b}$ Department of Solid-State Physics, National Research Centre (NRC), 12622 Cairo, Egypt

${ }^{c}$ Nanoscience and Technology Department, National Centre for Physics, 440000 Islamabad, Pakistan

${ }^{d}$ Department of Chemistry, University of Konstanz, 78467 Konstanz, Germany

${ }^{e}$ Department of Chemistry \& Zukunftskolleg, University of Konstanz, 78467

Konstanz, Germany

$\dagger$ Electronic supplementary information (ESI) available. See DOI: 10.1039/ d1ma00678a
}

function $(6.8-6.9 \mathrm{eV}){ }^{3}$ which can reduce the energy barrier at the $\mathrm{MoO}_{3} /$ absorber interface.

$\mathrm{MoO}_{3}$ is well known to possess a high density of oxygen vacancies, which are compensated by partially reduced $\mathrm{Mo}^{5+}$ and $\mathrm{Mo}^{4+}$ centres. ${ }^{4,5}$ Stoichiometric $\alpha-\mathrm{MoO}_{3}$ has a d ${ }^{0}$ configuration and is an electric insulator. These kinds of defects, which act as donor levels, are characteristic for all $\mathrm{d}^{0}$ oxides and play a considerable role in tuning the work function, following the linear Gordy-Thomas relation between the electronegativity and the work function. ${ }^{6}$ The electronic structure of $\mathrm{MoO}_{3}$ can be easily tuned by manipulating its stoichiometry via controlling the oxygen vacancies. ${ }^{7}$ This changes the defect density and thus the electronic properties such as energetics and conductivity. Herein, the atmospheric conditions play a key role. For example, $\mathrm{MoO}_{3}$ films when exposed to a hydrogen rich atmosphere at room temperature form molybdenum bronze. ${ }^{8}$ This introduces gap states in $\mathrm{MoO}_{3}$ and results in a decrease in the optical absorption above the bandgap. Similarly, a change in the work function was reported when $\mathrm{MoO}_{3}$ was exposed to reducing as well as oxidizing atmospheres. ${ }^{9}$ A seminal work by Deb suggested that the existence of oxygen vacancies is associated with different oxidation states such as $\mathrm{Mo}^{4+}$ or/and $\mathrm{Mo}^{5+} \cdot{ }^{10}$ Oxygen deficiency leads to the formation of color centers when electrons are trapped in the vacancy. Formation of these color centers is responsible for the chromogenic nature 
of $\mathrm{MoO}_{3}$, which describes the changes in the optical density and electronic properties caused by the oxygen deficiency. Color centers (F-centers) can form via UV illumination, ${ }^{11}$ thermal treatment and applying an electric field. ${ }^{12}$

Several theoretical ${ }^{13,14}$ and experimental ${ }^{15-17}$ studies have investigated the driving force behind the vacancy formation and their effects on the electronic properties of $\mathrm{MoO}_{3}$. A key challenge in modelling the vacancy mechanism is attributed to the layered structure of $\alpha-\mathrm{MoO}_{3}$ and the weak interaction between the layers. ${ }^{14}$ Experimentally, oxygen vacancy positions can be mapped via scanning transmission electron microscopy (STEM), ${ }^{15,16}$ X-ray photoelectron spectroscopy and other techniques. $^{17}$

In the present study, we combine both effects, the thermochromic effect and the sensitivity to oxidizing/reducing atmospheres in nanoblade structures, to tune the electronic properties. As far as we know, no previous accurate report on the lattice distortion mechanism on $\mathrm{MoO}_{3}$ had been established until the study by Ding et al. in $2014 .{ }^{18}$ Various van der Waals (vdW) corrected density function theories (DFT) have been applied to result in a decisive valuable study on lattice parameters, bond lengths and formation energies and deformation in $\mathrm{MoO}_{3} \cdot{ }^{19}$ Moreover, it was believed that $\alpha-\mathrm{MoO}_{3}$ is a p-type material until the last mentioned theoretical studies suggested it to be an n-type material. This study presents oxidized and reduced vertically aligned $\alpha-\mathrm{MoO}_{3}$ nanoblades on FTO substrates, which have been fabricated by annealing the films under different atmospheres $\left(\mathrm{O}_{2}, \mathrm{H}_{2}\right.$, vacuum and $\left.\mathrm{N}_{2}\right)$ at $450{ }^{\circ} \mathrm{C}$ for $3 \mathrm{~h}$. Subsequently, the positions of the oxygen vacancies and restitution of the oxygen ions in the $\mathrm{MoO}_{3}$ crystal have been experimentally determined primarily via electron paramagnetic resonance, high-resolution electron microscopy and other techniques. Our study reveals that the oxygen vacancies form within the bilayer in the $x$ - and $z$-planes. Furthermore, the impact of such intrinsic defects on the electrical-optical properties has been demonstrated.

\section{Results and discussion}

Structural and morphological analysis of reduced and oxidized vertically aligned $\alpha-\mathrm{MoO}_{3}$

Vertically aligned $\alpha-\mathrm{MoO}_{3}$ nanoblades of $5 \mu \mathrm{m}$ length grown on fluorine-doped tin oxide (FTO) substrates are treated under different oxidizing and reducing atmospheres (such as $\mathrm{O}_{2}, \mathrm{H}_{2}$, vacuum, and $\mathrm{N}_{2}$ ) at $400{ }^{\circ} \mathrm{C}$ for $3 \mathrm{~h}$. We note that a further increase in the annealing temperature beyond $450{ }^{\circ} \mathrm{C}$ leads to deformation of the nanoblades (see the ESI $\dagger$ Fig. S1). X-Ray diffraction (XRD) patterns of $\alpha-\mathrm{MoO}_{3}$ nanoblades after treatment under different oxidizing and reducing atmospheres are shown in Fig. 1a. The XRD patterns are consistent with orthorhombic $\alpha-\mathrm{MoO}_{3}$ (JCPDS: 00-005-508, space group Pbnm and

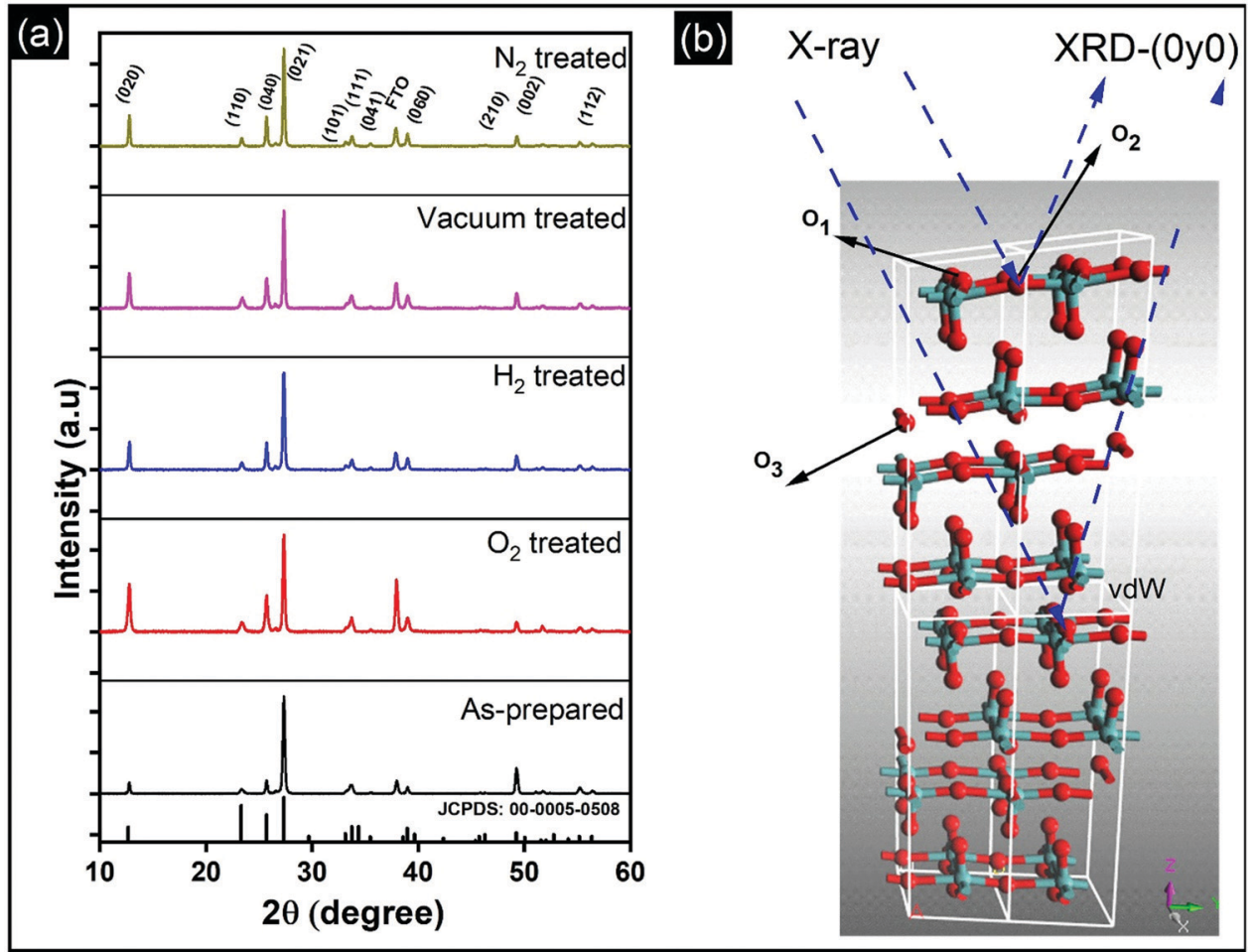

Fig. 1 (a) X-Ray diffraction patterns of vertically aligned $\alpha$-MoO3 on FTO substrates prepared by hydrothermal technique and post-treated at $400{ }^{\circ} \mathrm{C}$ for $3 \mathrm{~h}$ under different atmospheres such as oxidizing $\left(\mathrm{O}_{2}\right)$ or reducing $\left(\mathrm{H}_{2}\right.$, vacuum, $\left.\mathrm{N}_{2}\right)$. (b) Illustration of the crystal structure of the vertically aligned $\alpha-\mathrm{MoO}_{3}$ nanoblades grown in $z$-direction with indication of the stacked layers via van der Waals (vdW) bonds in the $y$-plane and its oxygen atom positions in the crystal as $\mathrm{O}_{1}, \mathrm{O}_{2}, \mathrm{O}_{3}$ in apical, corner sharing and edge sharing in the octahedra, respectively. The dashed blue lines illustrate the incident $\mathrm{X}$-ray beam on the substrates on the direction of the vdW bonds. 
unit cell parameters $a=13.86 \AA$, $b=3.70 \AA$ and $c=3.96 \AA$ ). ${ }^{20,21}$ The asymmetric oxygen lattice sites in the crystal are denoted as $\mathrm{O}_{1}, \mathrm{O}_{2}, \mathrm{O}_{3}$, where $\mathrm{O}_{1}$ is at the apex of the octahedra, $\mathrm{O}_{2}$ is at a corner-sharing position, and $\mathrm{O}_{3}$ is edge-sharing. The (vdW) interaction between adjacent layers is mediated via the apical oxygen atoms in the $y$-plane.

The diffraction patterns of the $\alpha-\mathrm{MoO}_{3}$ nanoblades treated at $400{ }^{\circ} \mathrm{C}$ under oxidizing and reducing atmospheres show the material to be highly crystalline; there were no peak shifts observed relative to the patterns of as-prepared films. However, we note that the peak intensities were significantly dependent on the treatment conditions. The origin of such a variation in the XRD peak intensity has been attributed in the literature to the change in the preferred crystal orientation, stoichiometry, and defects. A change of crystal orientation normally occurs due to the change in the synthesis process which is not applicable in our case. ${ }^{22,23}$ Table 1 summarizes the change in the relative intensity of the XRD diffraction peaks, which is calculated with respect to the (021) diffraction peak. Interestingly, the relative intensities increase in $(0 k 0)$ reciprocal planes with $k=2,4,6$. This reflects an increase in the number of scattering centers, which causes a more pronounced amplitude of the scattered intensity in that plane and suggests that none of the vacancies are introduced in the $y$-plane (van-der-Waals gap) $\left(\mathrm{O}_{1}\right.$ lattice site; see Fig. $\left.1 \mathrm{~b}\right)$. In contrast, the relative intensities in the (002) plane decrease, which suggests a decrease in the scattering centers (atoms, $\mathrm{O}_{3}$ lattice site) in that plane. The increase in the number of scattering centers in the $y$-direction is consistent with the work of Duo et al., which suggests that rearrangement and refilling of the vacancies spontaneously happens in the $y$-direction, ${ }^{24}$ affirming our understanding of the existence of the vacancies in the $(x, z)$ plane.

The increase in the peak intensity suggests an improved crystallinity under all conditions. According to vdW-DFT calculations by Inzani et al., the oxygen vacancies in the $\mathrm{O}_{1}$ and $\mathrm{O}_{3}$ lattice sites cause less lattice distortion in comparison to the $\mathrm{O}_{2}$ site where the highest lattice distortion can occur. ${ }^{17}$ The DFT + revised Perdew-Burke-Ernzerhof (RPBE) calculations by R. Tokarz further indicate that the $\mathrm{O}_{1}$ site $(y$-plane) has the largest formation energy in comparison to oxygen defects in the $\mathrm{O}_{2}$ and $\mathrm{O}_{3}$ sites. ${ }^{25}$ This implies that the oxygen vacancies in the $\mathrm{O}_{2}$ and $\mathrm{O}_{3}$ sites allow polaron migration $(T>20 \mathrm{~K})$ and

Table 1 A comparison of the change in the relative intensity (RI \%) of the XRD peaks with respect to the predominant peak (021) for $\alpha-\mathrm{MoO}_{3}$, asprepared and treated under $\mathrm{O}_{2}, \mathrm{H}_{2}$, vacuum, and $\mathrm{N}_{2}$ conditions

\begin{tabular}{lrrrrr}
\hline & \multicolumn{5}{c}{ Relative intensity with respect to $(021)$} \\
\cline { 2 - 6 } Plane & As-prepared & $\mathrm{O}_{2}$ & $\mathrm{H}_{2}$ & Vacuum & $\mathrm{N}_{2}$ \\
\hline$(021)$ & 100 & 100 & 100 & 100 & 100 \\
$(020)$ & 11 & 49 & 29 & 35 & 31 \\
$(110)$ & 4 & 9 & 7 & 11 & 8 \\
$(040)$ & 14 & 38 & 28 & 29 & 30 \\
$(111)$ & 9 & 14 & 10 & 13 & 10 \\
$(060)$ & 7 & 14 & 11 & 13 & 13 \\
$(002)$ & 26 & 10 & 14 & 14 & 10
\end{tabular}

replenishment of vacancies in the $\mathrm{O}_{1}$ sites. The polaron migration can occur by charges hopping between nonequivalent $\mathrm{Mo}^{6+}$ sites $(i, j)$, which assess the existence of $\mathrm{Mo}^{5+}$ and related oxygen vacancy, as given by the relation ${ }^{26}$

$$
\mathrm{Mo}_{i}^{6+}+\mathrm{Mo}_{j}^{5+} \stackrel{\Delta G^{\ddagger}}{\rightarrow} \mathrm{Mo}_{i}^{5+}+\mathrm{Mo}_{j}^{6+}
$$

To gain further insights into vacancy formation and to validate the XRD data, we performed high resolution transmission electron microscopy (HR-TEM) and recorded selected area electron diffraction (SAED) patterns of the $\alpha-\mathrm{MoO}_{3}$ nanoblades. SAED has been used to define dislocations and deformations in the diffracted lattice points (corresponding to the lattice spacing $d$ ) in the $x$ - and $z$-planes (Fig. 2). The surfaces of all posttreated $\alpha-\mathrm{MoO}_{3}$ nanoblades show random spots of lower contrast after the heat treatment for all conditions. The SAED patterns show no deviation from the initial degree of crystallinity after various treatments, as also confirmed by XRD data. Additionally, the SAED patterns reflect the diffraction patterns corresponding to the zone axis of $[x 0 z]$. This allows us to calculate the inter-planar spacing ( $d$-spacing) in those planes ( $d=2 /$ reciprocal lattice). The $d$-spacing values (Table 2 ) remain nearly unchanged in one direction and are in good agreement with the standard lattice constant $a=0.396 \mathrm{~nm}$ ( $x$-direction). In the $z$-direction, the $d$-spacing values, however, vary significantly from the standard (lattice constant $c=0.369 \mathrm{~nm}$ ). The $d$-spacing value for the $\mathrm{O}_{2}$-treated sample shows the best match with the standard value, suggesting that oxygen treatment tends to produce highly defect-free nanoblades. The $d$-spacing values for the reduced $\alpha-\mathrm{MoO}_{3}$ samples show a higher deviation from the standard value. The vacuum treated samples show a smaller deviation, while the $\mathrm{H}_{2}$ and $\mathrm{N}_{2}$ treated samples show the highest deviations.

These results suggest that the oxygen vacancies are introduced into the crystal upon thermal post-treatment. According to vdW-DFT calculations, the formation of oxygen vacancies in the $z, x$-plane leads to less perturbation (not exceeding $\approx 0.02 \mathrm{~nm}$ ) in the positions of Mo ions toward the closest $\mathrm{O}$ ions in comparison to $y$-plane oxygen vacancies. ${ }^{18}$ This closely matches the $d$-spacing values of $\mathrm{O}_{2}$ and vacuum-treated samples. In the case of $\mathrm{H}_{2}$ and $\mathrm{N}_{2}$ treated samples, however, we note a deviation $>0.02 \mathrm{~nm}$. We attribute this to substitution of the oxygen atom for the adsorbed $\mathrm{H}$ and $\mathrm{N}$, which can be assumed as an external doping (defect), respectively. The substitution of an oxygen atom (atomic radius $0.48 \AA^{27}{ }_{i}$ ) with the larger atomic $\mathrm{H}$ and $\mathrm{N}$ atoms (atomic radii 0.53 and $0.56 \AA$, respectively) can be the cause of the additional shrinking of the $d$-spacing, as a relatively small increase of the cell volume and slight distortion of the lattice, ${ }^{8}$ see Table 2 .

We further employed electron paramagnetic resonance (EPR), which is a sensitive tool for detecting a minor concentration of shear structures, point defects, reduced ions, oxygen radical species, and colour centers. ${ }^{28}$ Fig. 3a shows the continuous wave (CW) EPR spectra recorded at $80 \mathrm{~K}$ of as-prepared $\alpha-\mathrm{MoO}_{3}$ nanoblades and of $\alpha-\mathrm{MoO}_{3}$ nanoblades treated under oxidizing and reducing atmospheres. The signal intensity is 

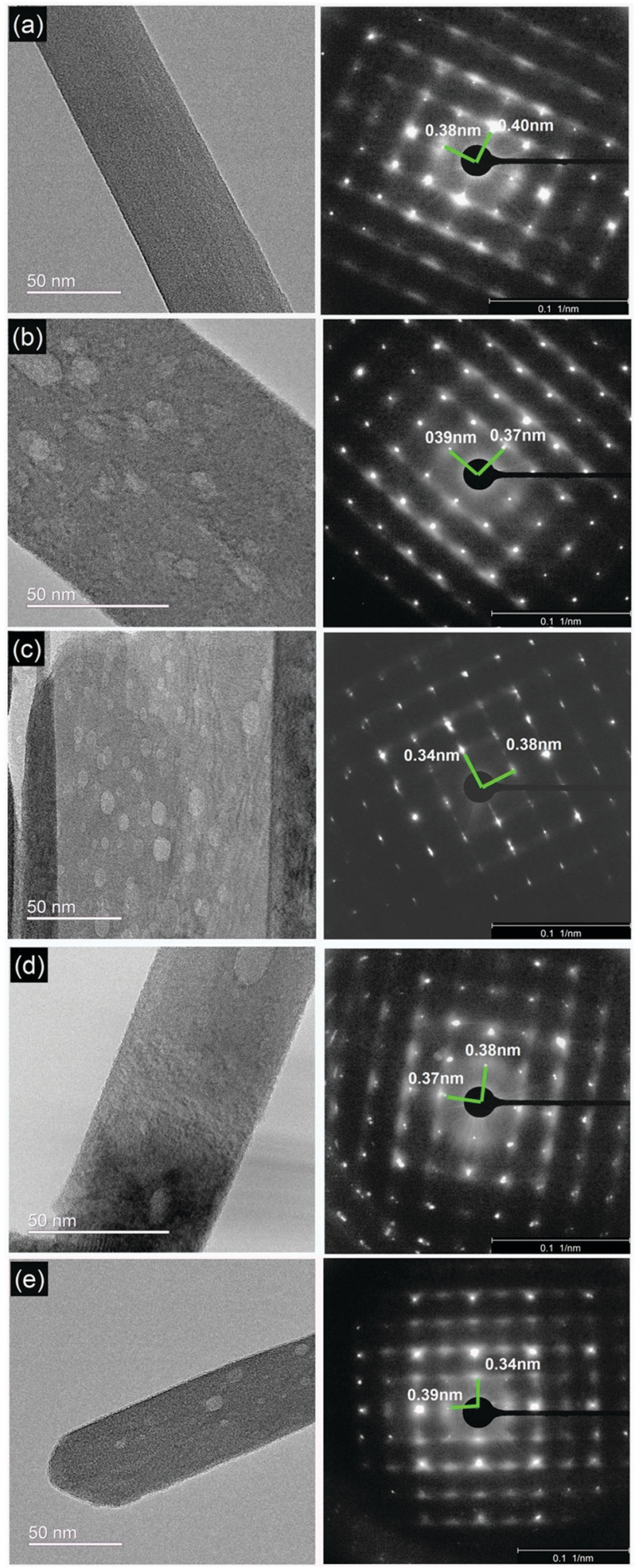

Fig. 2 (a-e) HR-TEM micrographs and SAED patterns of as-prepared $\alpha-\mathrm{MoO}_{3}$ nanoblade and treated nanoblades under $\mathrm{O}_{2}, \mathrm{H}_{2}$, vacuum and $\mathrm{N}_{2}$ conditions, respectively with the measured $d$-spacings.

plotted as a function of the $g$ values. This allows for a direct comparison of the EPR spectra irrespective of slight variations
Table 2 The $d$-spacing values of the as-prepared and post-treated $\alpha$ $\mathrm{MoO}_{3}$ nanoblades under $\mathrm{O}_{2}, \mathrm{H}_{2}$, vacuum, and $\mathrm{N}_{2}$ conditions

\begin{tabular}{lll}
\hline & Lattice-spacing $(\mathrm{nm})$ & \\
\cline { 2 - 3 } Samples & $\mathrm{dc}$ & $\mathrm{da}$ \\
\hline As-prepared & 0.38 & 0.40 \\
$\mathrm{O}_{2}$ & 0.37 & 0.39 \\
$\mathrm{H}_{2}$ & 0.34 & 0.38 \\
Vacuum & 0.37 & 0.38 \\
$\mathrm{~N}_{2}$ & 0.34 & 0.39
\end{tabular}

of the microwave frequency from one measurement to the other. The $g$-values have been calculated according to the following equation: ${ }^{29}$

$$
g=\frac{h \nu}{\mu_{\mathrm{B}} B_{0}}
$$

where $h \nu$ is the microwave energy, $\mu_{\mathrm{B}}$ the Bohr magneton, and $B_{0}$ the applied magnetic field. EPR transitions appear at the $g$-values typical for $\alpha-\mathrm{MoO}_{3}$ materials. The broad signals at around $g=1.94$ are attributed to $\mathrm{Mo}^{5+}$ species. ${ }^{30,31}$ The hyperfine coupling owing to isotopes with $I=5 / 2$ (natural abundance for ${ }^{95} \mathrm{Mo}$ and ${ }^{97} \mathrm{Mo}$ is $15.92 \%$ and $9.55 \%$, respectively) is not resolved. ${ }^{32,33}$ Qualitative inspection of the spectral line shape suggests that the samples can be grouped by similarities of their EPR signals as follows: $\mathrm{H}_{2}$ and $\mathrm{N}_{2}$, as-prepared and vacuum, and $\mathrm{O}_{2}$. The EPR signals are significantly broader than those expected for individual $\mathrm{Mo}^{5+}$ species, which can be an indication of the degree of reduction. ${ }^{28,30}$ The integrated intensity normalized to the sample weight is the largest for $\mathrm{H}_{2}$ and $\mathrm{N}_{2}$; it is by a factor of 10 smaller for as-prepared and vacuum treated nanoblades, and almost zero for oxygen treated nanoblades as can be seen in Fig. 3c. The integrated intensity of these EPR spectra reflects the number of $\mathrm{Mo}^{5+}$ ions in the nanoblades. The broadening of the peaks suggests that they consist of several overlapping signals, which is in line with previously reported studies. ${ }^{28,30,31,34,35}$ The lower values of the absorption g-tensor scalar around 2.003, which were detected in reduced samples $\left(\mathrm{H}_{2}\right.$, vacuum, $\left.\mathrm{N}_{2}\right)$, can be ascribed to single ionized oxygen vacancies $\mathrm{O}^{\bullet-}$ (radical oxygen), ${ }^{35}$ see Fig. $3 \mathrm{~b}$.

In oxidized $\alpha-\mathrm{MoO}_{3}$ nanoblades a low intensity signal has been recorded, which is at the same position of the original signal of $\alpha-\mathrm{MoO}_{3}$. This signal can be attributed to a very low concentration of $\mathrm{Mo}^{5+}$ ions and the existence of weakly bound F-centers. The F-centers have been assumed to exist as metal cations in two different oxidation states $(6+$ and $5+)$, where the free electron (stemming from oxygen vacancies) can be trapped into other cation sites. Pick et al. have shown the existence of two types of F-centers, which differ in how strongly the electron is bound to the defect site. The weakly bound centers can be ionized and bleached at low temperatures with a broad absorption band. ${ }^{36,37}$ The more strongly bound F-center requires a higher temperature to be ionized and provides electrons to the weakly bounded F-center as a bound excited state. ${ }^{38}$ The spectrum of vacuum treated nanoblades exhibits an asymmetric line shape, which is attributed to the local crystal field 


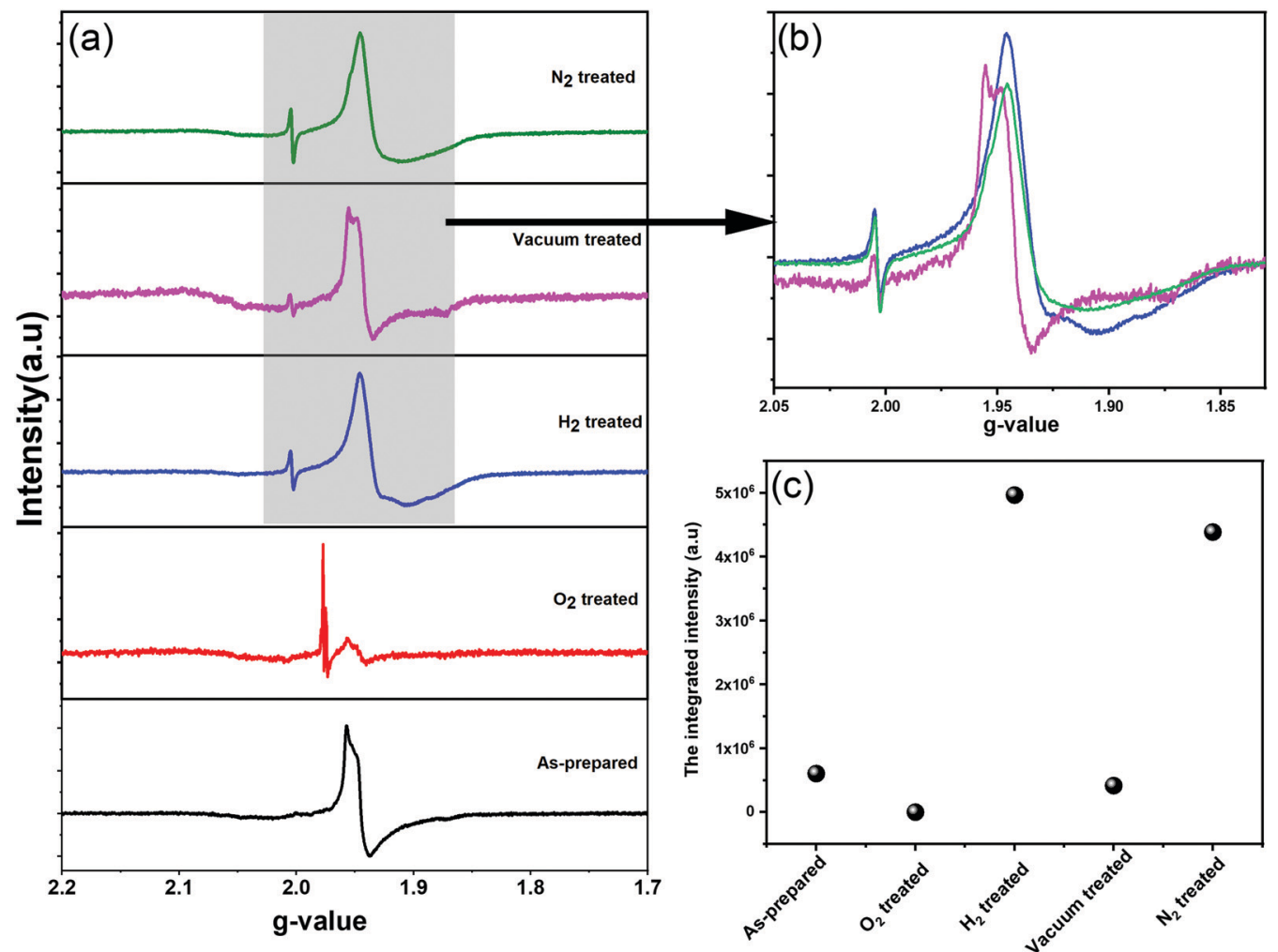

Fig. 3 (a and b) EPR spectra of as-prepared $\alpha-\mathrm{MoO}_{3}$ nanoblades and treated nanoblades under $\mathrm{O}_{2}, \mathrm{H}_{2}$, vacuum and $\mathrm{N}_{2}$ atmospheres. (c) The integrated intensity versus $\alpha-\mathrm{MoO}_{3}$ nanoblades treatment condition.

with nonaxial symmetry and a pronounced hyperfine structure, in agreement with previous studies. ${ }^{28,30-32}$ However, in the $\mathrm{N}_{2}$ treated nanoblades the hyperfine structure cannot be observed. The $\mathrm{H}_{2}$ treated nanoblades show the same broadening as the ones treated with $\mathrm{N}_{2}$, with a hyperfine structure related to g-tensors that fits well with previous reports on the effect of $\mathrm{H}^{+}$on the $\mathrm{MoO}_{6}$ octahedral structure. Untreated and reduces $\alpha-\mathrm{MoO}_{3}$ nanoblades g-tensors are found to be 1.956 and 1.946, respectively, and reflect $\mathrm{Mo}^{5+}$ species which can be explained by structural changes. ${ }^{39}$ Various previous EPR studies of $\mathrm{Mo}^{5+}$ species assigned that signal to hexacoordinated $\mathrm{Mo}(\mathrm{V})$ centers in shear defects caused by an off-center shift of the Mo atom towards a surrounding oxygen. ${ }^{30,40}$ Those results suggest a rearrangement of the vacancies from corner to edge sharing ( $x$ - to $z$-) planes, in agreement with the finding of a decrease in the relative intensity of the z-plane in XRD patterns. Additionally, the spin Hamiltonian function which has been given by K. Dyrek et al., indicates a weakening of the octahedral crystal field around $\mathrm{Mo}^{5+}$, which confirms a strong lattice distortion in that plane. ${ }^{30}$ A hydrogen rich atmosphere is found to reduce $\mathrm{Mo}^{6+}$ and result in full occupancy of the anionic sites addressed to the $\left(\mathrm{MoO}_{6}\right)^{7-}$ cluster of octahedral $\mathrm{Mo}^{5+}$. $\mathrm{A} \mathrm{N}_{2}$ rich atmosphere can accept one electron from the oxygen in the crystal and result in $\mathrm{N}_{2}{ }^{-}$(not paramagnetic active impurities), which allows detection of stabilized radical $\mathrm{O}^{\bullet-}$ in shear structures along the $x, z$-plane. ${ }^{28,32}$ The lower intensity of the vacuum treated sample spectrum confirms the lower concentration of free radical ions, which can lead to a difference in the electrical conductivities between $\mathrm{N}_{2}, \mathrm{H}_{2}$ and vacuum treated samples. It is essential to indicate that $\mathrm{Mo}^{4+}\left(\mathrm{MoO}_{2}\right)$ can be formed in both cases of rich $\mathrm{H}^{+}$and high-temperature vacuum treated conditions (have not been detected by XRD) or even $\mathrm{Mo}^{3+}$ (not sensitive to EPR). For oxidized $\mathrm{MoO}_{3}$ nanoblades, the EPR signal for the radical $\mathrm{O}^{2-}$ stabilized in $\mathrm{Mo}^{6+}$ was not detected in our case, which suggests the higher stability of our structure (ex situ nanoblades).

Using XRD, SAED, and EPR we have verified that oxygen vacancies have been eliminated from corner to edge-sharing $\alpha-\mathrm{MoO}_{3}$ nanoblades ( $x$-, $z$-planes). A quantity of $\mathrm{Mo}^{5+}$ ions in each sample was determined from the comparison with the EPR intensity of a standard $\mathrm{CuSO}_{4}$ sample as an indication of substoichiometric effects. Under the assumption that only $\mathrm{Mo}^{5+}$ species contribute to the EPR intensity and that there is an oxygen vacancy corresponding to each $\mathrm{Mo}^{5+}$ ion, the stoichiometry of the $\alpha-\mathrm{MoO}_{3}$-based materials was evaluated in Table 3. To identify the paramagnetic species/defects in the oxidized and reduced $\alpha-\mathrm{MoO}_{3}$ nanoblades, we undertook spectral simulations (ESI $\dagger$ Fig. S2). Due to the broad EPR spectra that lack any resolved fine structure, the characteristic $g$-values of individual $\mathrm{Mo}^{5+}$ species cannot be extracted from the spectrum. Instead, a set of known $g$-values was used, which corresponds to various individual $\mathrm{Mo}^{5+}$ species as they have been determined in reduced $\alpha-\mathrm{MoO}_{3}$ nanoblades.

\section{Electrical characterization of $\alpha-\mathrm{MoO}_{3}$ nanoblades}

To measure the electronic properties, vertical $\alpha-\mathrm{MoO}_{3}$ nanoblade films have been connected between two metal electrodes 
Table 3 The estimated stoichiometry evaluation of as-prepared $\alpha-\mathrm{MoO}_{3}$ nanoblades, and of nanoblades treated under oxidizing $\left(\mathrm{O}_{2}\right)$ or reducing atmospheres $\left(\mathrm{H}_{2}\right.$, vacuum, $\left.\mathrm{N}_{2}\right)$ using EPR

\begin{tabular}{ll}
\hline Sample & Stoichiometry \\
\hline As-prepared & $\mathrm{MoO}_{2.994}$ \\
$\mathrm{O}_{2}$ treated & $\mathrm{MoO}_{3.000}$ \\
$\mathrm{H}_{2}$ treated & $\mathrm{MoO}_{2.951}$ \\
Vacuum treated & $\mathrm{MoO}_{2.996}$ \\
$\mathrm{~N}_{2}$ treated & $\mathrm{MoO}_{2.957}$ \\
\hline
\end{tabular}

as a bottom-top electrode configuration. Fig. 4a shows the FE-SEM top micrograph of evaporated $\mathrm{Au}$ on the vertically aligned $\alpha-\mathrm{MoO}_{3}$ nanoblades on FTO substrates. Room temperature current-voltage $(I-V)$ characteristic curves of as-prepared and thermally treated $\mathrm{Au} / \mathrm{MoO}_{3}$ nanoblades on FTO substrates are shown in Fig. $4 \mathrm{~b}$.

The electric field through such an electrode structure is not uniform (see Fig. 4c). These electrodes, however, offer a higher current sensitivity in comparison to conventional rectangular electrodes. ${ }^{41}$ In addition, it has been demonstrated that the narrow gap between the electrode terminals leads to a lateral current flow. This is different from the conventional co-planar electrodes (with a larger inter-electrode gap), where a lateral and horizontal current flow is likely (perpendicular to the substrates) (Fig. 4d). ${ }^{42}$ In our electrode setup, the current can flow along two possible pathways, directly to the second terminal electrode via interconnected nanoblades or via the seed layer (see Fig. 4d). The seed layer shows a nearly insulating behaviour at room temperature (Fig. S3a, ESI $\dagger$ ), consistent with previous reports on the air treated $\alpha-\mathrm{MoO}_{3}$ as the insulator layer. ${ }^{43,44}$

We recorded temperature dependent $I-V$ characteristics of $\alpha-\mathrm{MoO}_{3}$ devices (Fig. S4a-e, ESI $\dagger$ ). The semi-logarithmic temperature dependent $I-V$ curves show nonlinearity and symmetric trends at both forward and reverse biasing conditions. This behavior suggests the formation of a negligible Schottky barrier at the interfaces between the metal electrode and the nanoblades ( $\mathrm{Au} / \alpha-\mathrm{MoO}_{3} / \mathrm{Au}$ diode). ${ }^{45,46}$ The non-linearity in the $I-V$ characteristics suggests the formation of the barrier at the interfaces between the electrodes and the metal oxide. ${ }^{45}$ The conduction mechanism shows Ohmic conduction (0-3 V), which is categorized as a bulk limited conduction mechanism. Such a conduction mechanism shows dependence on the intrinsic semiconductor properties resulting from the movements of mobile electrons in the conduction bands and holes in the valence band. ${ }^{47}$ The electrical conductivity $(\sigma)$ has been calculated in the Ohmic region using the following relation: ${ }^{47}$

$$
J=\sigma E=q \mu N_{\mathrm{C}} E \exp \left\lfloor\frac{-\left(E_{\mathrm{C}}-E_{\mathrm{f}}\right)}{k T}\right\rfloor
$$

The activation energy $E_{\mathrm{A}}$ can be deduced from the Arrhenius law as follows:

$$
\sigma=\sigma_{0} \exp \left(\frac{-\left(E_{\mathrm{C}}-E_{\mathrm{f}}\right)}{k T}\right)=\sigma_{0} \exp \left(\frac{-E_{\mathrm{A}}}{k T}\right)
$$

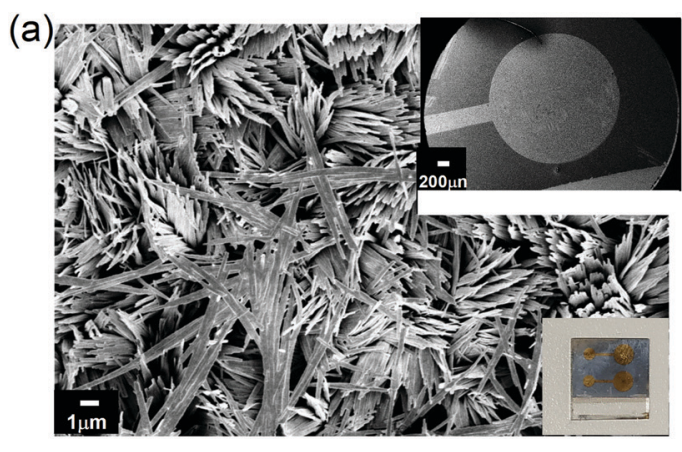

(c)

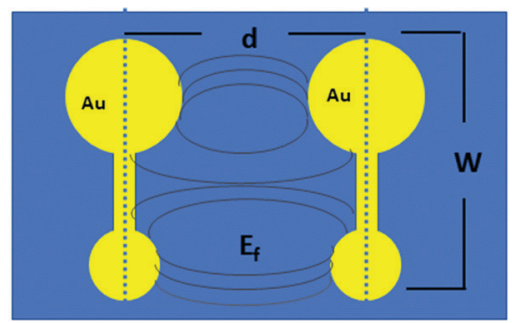

(b)

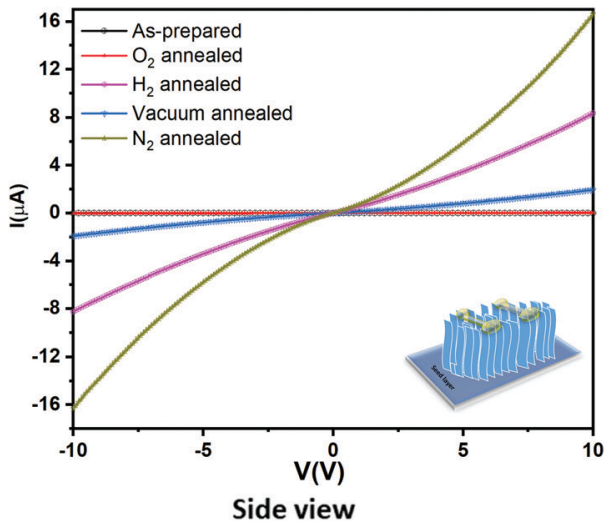

(d)

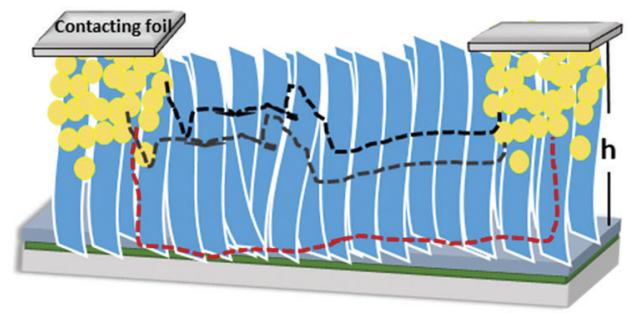

Fig. 4 (a) FE-SEM micrograph of evaporated Au on the vertically aligned $\alpha-\mathrm{MoO}_{3}$ nanoblades; the inset is a schematic resembling a final electrical device. (b) Room temperature $I-V$ curves of as-prepared post-treated $\alpha-\mathrm{MoO}_{3}$ nanoblade based devices. (c) Top view of the evaporated co-planar Au electrodes ( $d$ is the distance between the two central points of the electrodes terminals, $W$ is the length of the electrode, $E_{f}$ is a schematic of the supposed electrical field). (d) Side view of the electrical device ( $h$ is the nanoblade length) with illustration of possible electrical pathways through the devices. The red dashed line represents charge flow through the nanoblades and the seed layer, and the black dashed line represents the path through interconnected nanoblades. 
Table 4 Room-temperature electrical conductivity, activation energy $\left(E_{\mathrm{A}}\right)$, and hopping activation energy ( $\left.E_{\text {hopping }}\right)$ in the applied voltage region of $0-3 \vee$ for as-prepared vertically aligned $\alpha-\mathrm{MoO}_{3}$ nanoblades and films treated under $\mathrm{O}_{2}$, vacuum, $\mathrm{H}_{2}$, and $\mathrm{N}_{2}$ rich atmospheres

\begin{tabular}{llll}
\hline Sample & Conductivity $\left(\mathrm{Sm}^{-1}\right)$ & $E_{\mathrm{A} 1}(\mathrm{eV})$ & $E_{\text {hopping }}(\mathrm{eV})$ \\
\hline As-prepared & $(6.04 \pm 2.85) \times 10^{-6}$ & $0.34 \pm 0.02$ & $0.38 \pm 0.05$ \\
$\mathrm{O}_{2}$ treated & $(3.67 \pm 0.90) \times 10^{-4}$ & $0.38 \pm 0.02$ & $0.41 \pm 0.02$ \\
$\mathrm{H}_{2}$ treated & $(9.95 \pm 2.57) \times 10^{-2}$ & $0.23 \pm 0.01$ & $0.24 \pm 0.02$ \\
Vacuum treated & $(2.60 \pm 0.33) \times 10^{-2}$ & $0.26 \pm 0.01$ & $0.29 \pm 0.00$ \\
$\mathrm{~N}_{2}$ treated & $(12.80 \pm 1.79) \times 10^{-2}$ & $0.28 \pm 0.01$ & $0.30 \pm 0.01$
\end{tabular}

$J$ is the current density (equal to the applied current $I$ divided by the contact area $A=3.5 \times 10^{-8} \mathrm{~m}^{2}$ (calculated by multiplying the total length of the electrode $W=8.71 \times 10^{-3} \mathrm{~m}$ with the height of the film $h=4 \times 10^{-6} \mathrm{~m}$ )), $E$ is the electric field (equal to the applied voltage divided by the average electrode separation distance $d$ of $\left.4.67 \times 10^{-3} \mathrm{~m}\right),{ }^{48,49} q$ is the electric charge, $\mu$ is the electron mobility, $N_{\mathrm{C}}$ is the effective density of states in the conduction band, and $E_{\mathrm{f}}$ is the Fermi energy level. The activation energies give the position of the Fermi level below the conduction band and have been calculated for all conditions using eqn (4) and are listed in Table 4.

Our vertically aligned $\alpha-\mathrm{MoO}_{3}$ films show significantly higher electrical conductivity in comparison to previous studies on similar nanostructured films based on $\alpha-\mathrm{MoO}_{3} \cdot{ }^{17,50}$ The $\mathrm{N}_{2}$, $\mathrm{H}_{2}$ and vacuum treated samples show a higher conductivity than their as-prepared counterparts. As expected, $\mathrm{O}_{2}$ treated nanoblades show a reduced conductivity, which confirms a readsorption and restitution of oxygen into the crystal. It is important to note that due to their highest conductivity, the $\mathrm{N}_{2}$ treated samples show a sudden decrease in the current at high temperature (Fig. S4e, ESI $\dagger$ ), which can be attributed to damage or deformation in the structures. The high conductivities can be attributed to different factors: the higher density of the interconnected nanoblades grown on the substrate facilitate the carrier transport, ${ }^{17}$ a high surface to volume ratio of the very thin sheet-like structures, and the crystalline nature of the nanoblades. ${ }^{17}$

The calculated values of $E_{\mathrm{A}}$ (Table 4 ) for the reduced and oxidized vertically aligned $\alpha-\mathrm{MoO}_{3}$ nanoblades imply the existence of shallow levels close to the conduction band minimum. These values match the previously reported theoretical and experimental values in the literature. ${ }^{14}$ The vdW-DFT calculations ${ }^{17}$ showed the transition energies of the three different oxygen vacancy positions to the valence band maximum to follow the order $\mathrm{O}_{1}<\mathrm{O}_{3}<\mathrm{O}_{2}$ (see Fig. $1 \mathrm{~b}$ for $\mathrm{O}$ positions in the crystal). The $\mathrm{O}_{1}$ vacancy constitutes the deepest intra-band level, whereas the $\mathrm{O}_{2}$ vacancy marks the shallowest defect state, as indicated in a recent study by Inzani et al. ${ }^{14} \mathrm{~A}$ further study by Ding et al. ${ }^{18}$ on the localized electron small polarons (refers to $\mathrm{Mo}^{5+}$ ) in $\alpha-\mathrm{MoO}_{3}$, revealed polaron migration and hopping to neighboring Mo sites in the same plane. Ding et al. highlighted anisotropy in the activation energy and hopping paths and distances within the same plane of the bilayer, zigzag in comparison to transition across the van der Waals gap. The lowest activation energy and fast polaron migration was found to be in the same plane of the bilayer sheet (corresponding to $\mathrm{O}_{2}, \mathrm{O}_{3}$ in the $(x, z)$ plane).$^{18}$ As previously demonstrated studies about the color center formation in $\mathrm{MoO}_{3}$, it can be explained via small polaron theory. ${ }^{18,51}$ The first investigation by Mott in $1968^{52}$ affirms that small polaron hopping occurs when thermal energies are coinciding with the closer energy level of nearby atoms, which contribute at the transition between different metal oxidation states. ${ }^{53}$ The conductivity is attributed to the movement of the electron between various cation sites, which suggests a hopping mechanism. ${ }^{52}$

The hopping activation energy $E_{\text {hopping }}$ can be determined through the following eqn (3): $:^{52}$

$$
\sigma T=\sigma_{00} \exp \left(-\frac{E_{\mathrm{hopping}}}{k T}\right)
$$

where $\sigma_{00}$ is the Mott constant for the hopping mechanism related to transition metal oxides (for more information $\mathrm{see}^{52}$ ). The inset of Fig. 5 shows a good fit of $\ln (\sigma T)$ versus $1 / T$ to the small polaron model. It is noteworthy that $E_{\text {hopping }}$ at high temperatures reflects not only the polaron hopping energy but also the disorder energy. This can explain the higher $E_{\mathrm{A}}$ via the hopping mechanism (see eqn (1)) compared to the ones obtained from the Arrhenius plot. Additionally, the higher values of the hopping energies are correlated with the activation energy from the extrinsic Fermi level concurring with the Mott model. The small addition in the hopping related energies in comparison to the calculated activation energies can be attributed to the average change in the energy within the movements between one cation site and another. ${ }^{52}$ The obtained activation energies of the reduced vertically aligned $\alpha-\mathrm{MoO}_{3}$ nanoblades $\left(\mathrm{H}_{2}\right.$, vacuum, and $\left.\mathrm{N}_{2}\right)$ agree well with theoretical calculations by Ding et al. ${ }^{18}$ which confirm polaron migration in the $x-z$-plane. However, the higher values of the oxidized $\alpha-\mathrm{MoO}_{3}$ nanoblades may suggest polaron migration across the zigzag path. Subsequently, the existence of shallow levels as intrinsic defects even in the oxidized vertically aligned

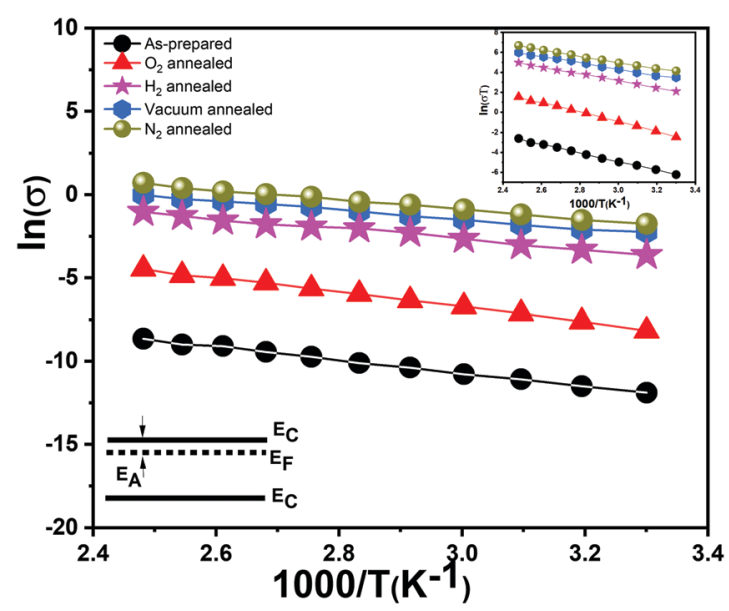

Fig. 5 Arrhenius plot on the Ohmic region of the applied voltage $(0-3 \mathrm{~V})$ with schematic illustrating the corresponding activation energy in the band diagram. The inset figure shows the Arrhenius plot corresponding to the hopping mechanism in the same region of the applied voltage. 
$\alpha-\mathrm{MoO}_{3}$ nanoblades confirms its n-type nature in the stoichiometric form, and degenerated n-type under reducing atmospheres. $^{24}$

\section{Optical characterization of $\alpha-\mathrm{MoO}_{3}$ nanoblades}

In order to investigate the role of oxygen vacancies in the color center formation, we performed diffuse reflectance spectroscopy (DRS). DRS can provide information about the dispersion and optical constants. DRS is considered as a reliable measurement for such film surfaces where multiple reflections and diffusion of the incident waves are likely to occur (porous-like surface of the vertical nanoblades). Recently, Kumar et al. ${ }^{54}$ measured various thick films using the diffuse reflectance to estimate the optical band gap using the relation ${ }^{55,56}$

$$
\frac{h \nu}{2 d} \ln \left(\frac{R_{\max }-R_{\min }}{R-R_{\min }}\right)=k\left(h \nu-E_{\mathrm{g}} \pm E_{\mathrm{p}}\right)^{1 / n}
$$

The optical band gap $E_{\mathrm{g}}$ is given by the McLean relation:

$$
\alpha(h \nu)_{\mathrm{ph}}=k\left(h \nu-E_{\mathrm{g}} \pm E_{\mathrm{p}}\right)^{1 / n}
$$

where $R_{\max }$ and $R_{\min }$ are the maximum and minimum reflectance in the reflectance spectrum, $R$ is the entire diffuse reflectance spectrum, and $d$ is the film thickness, $(h \nu)_{\mathrm{ph}}$ is the incident photon energy, the exponent $n$ is related to the nature of the transition ( $n=2$ for allowed direct, $n=2 / 3$ forbidden direct transition, $n=1 / 2$ allowed indirect transition, $n=1 / 3$ forbidden indirect transition), $E_{\mathrm{p}}$ is the shallow trap level energy (allowed direct transition) or phonon assisted transition (allowed indirect transition). Fig. 6a shows diffuse reflectance versus the incident wavelength. The film's color appearance (Fig. 6a, bottom inset) shows the thermochromic effect well known in $\mathrm{MoO}_{3} \cdot{ }^{57,58}$ There is a limitation for defining the exact F-center bands due to broadening in the peak, which was reported previously by Dexter due to the lattice vibrations. ${ }^{58}$ The spectra in Fig. 6a identify the F-center absorption (as bands 1 and 2), which occur in the incident wavelength range of 585$650 \mathrm{~nm}$ and 650-700 nm, respectively. Experimentally, two color center bands have been detected. The first one is related to weakly bound states with lower ionization energy, which can be stabilized at low temperatures. The other band has bound excited carriers, which requires high temperature for stabilization. As given by Pick et al., ${ }^{59}$ the F-center is considered to comprise two electrons trapped at an anion vacancy, which was confirmed through photoconductivity measurements by Domanic. ${ }^{38}$ This corroborates the existence of F-centers in the $\mathrm{O}_{2}$ treated sample and matches our electrical analysis very well. F-center bands for as-prepared and thermally post-treated films under vacuum positions are barely distinguishable. This observation reveals significant ramping in the colors for the films from bleaching white for oxygen treated to dark blue for the $\mathrm{N}_{2}$ treated film, which is correlated with stoichiometric and deficient vertically aligned $\alpha-\mathrm{MoO}_{3}$ nanoblades, respectively.

Fig. $6 \mathrm{~b}$ shows the allowed direct and indirect transitions. The lowest energy transition occurs as an indirect transition with an energy of $3.00 \pm 0.05 \mathrm{eV}$, and the direct transition happens at a slightly higher energy of $3.20 \pm 0.05 \mathrm{eV}$. This shows exciton absorption at an energy of $\approx 0.20 \mathrm{eV}$. We do not observe any significant change in the optical band gap; however, there is a minor change in the position of the color center bands and small variations between the concentrations of the color center under different reducing atmospheres in comparison to treatment under a rich $\mathrm{O}_{2}$ atmosphere which shows the lowest density. The color center density $N\left(\mathrm{~cm}^{-3}\right)$ can be estimated through Smakula's equation: ${ }^{60}$

$$
N f=C \cdot 10^{17} \cdot \frac{n}{\left(n^{2}+2\right)^{2}} \cdot \alpha u
$$

where $C$ is a factor depending on the shape of the band (1.29 in the case of approximately Gaussian and 0.89 in the case of Lorentzian shape), $f$ is the oscillator strength (between 0.1 and 1), $n$ is the refractive index $=2,^{58} \alpha$ is the absorption coefficient at that band (in $\mathrm{cm}^{-1}$ ) and $u$ is the full width at half maximum of that band (in eV). The oscillator strength has a classical and quantum mechanical explanation, which can involve the expression of the polarizability of the atoms. ${ }^{57}$ There is no simple way to determine the absolute value of $N$ (a)

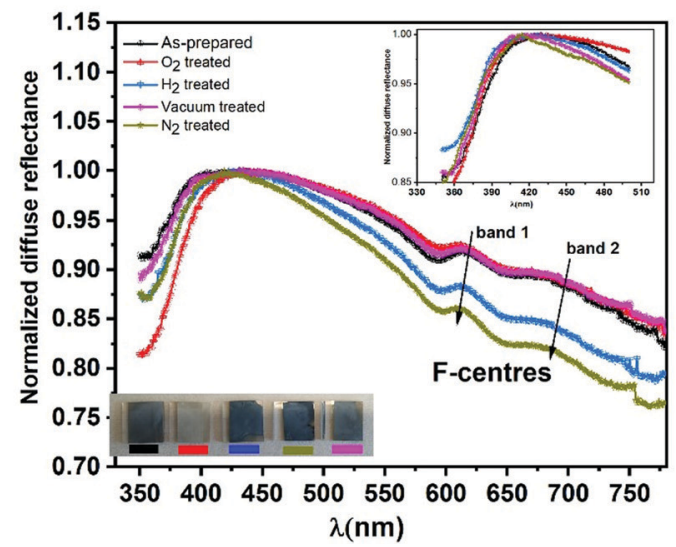

(b)

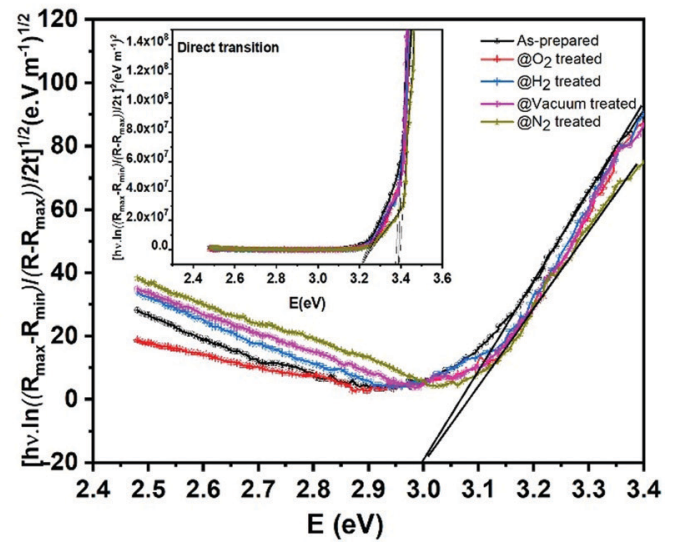

Fig. 6 (a) Diffuse reflectance spectra of vertically aligned $\alpha-\mathrm{MoO}_{3}$ on FTO substrates as-prepared and treated under $\mathrm{O}_{2}, \mathrm{H}_{2}$, vacuum and $\mathrm{N}_{2}$ atmosphere, the inset figure shows the optical bandgap position. (b) Direct and indirect transitions subtracted from diffuse reflectance spectra. 
due to the uncertainty in the band FWHM and the overlap of the bands. Subsequently, the average values of density of F-centers and oscillator strength have been given in Table S1 (ESI $\dagger$ ), which correspond to their central band energy. The obtained values are slightly lower than previous studies, ${ }^{57}$ which reflects lower defect densities corresponding to not strongly colored samples. The density colour center for bands 1 and 2 for the treated sample under an $\mathrm{O}_{2}$ atmosphere shows lower values in comparison to other treated samples under reduced atmospheres $\left(\mathrm{H}_{2}\right.$ and $\left.\mathrm{N}_{2}\right)$. This suggests the higher and faster reduction influence by those atmospheres in comparison to vacuum treated samples. The corresponding absorption to band 1 suggest consistent behavior with F-centers (thermodynamic stable center) as given by $\mathrm{D}$. Lynch et al. ${ }^{38}$ which demonstrates the existence of weakly bound $F$-centres, easily thermally ionized states as a result of F-center formation. The weakly bound F-centers suggest that there is no absolute stoichiometric $\alpha-\mathrm{MoO}_{3}$ until the material is cooled down to below $20 \mathrm{~K}$ to stabilize those weakly bound states. In contrast, according to band 2, there is a statistical distribution for all the cases, which has a good correlation with the data obtained by EPR measurements.

\section{Conclusions}

Vertically aligned $\alpha-\mathrm{MoO}_{3}$ nanoblades have been introduced into oxidizing and reducing atmospheres at a temperature of $400{ }^{\circ} \mathrm{C}$ to tune their oxygen vacancy concentrations. Our results demonstrate a fundamental experimental approach for the formation of oxygen vacancies within the bilayer of the crystal structure, which is consistent with previously reported theoretical and computational studies. The amount of the oxygen deficiencies in $\alpha-\mathrm{MoO}_{3}$ nanoblades as a result of the posttreatment under oxidizing and reducing atmospheres has been evaluated using EPR. The electronic properties have been studied using top co-planar electrodes. The material shows n-type behavior under reducing atmospheres and degenerated n-type behavior under an oxidizing atmosphere. This has been explained using color-center formation models and polaron migration. The electrical conductivity is found to be altered in the change from $7 \times 10^{-6}$ to $1 \times 10^{-2} \mathrm{Sm}^{-1}$ for oxidized to reduced $\alpha-\mathrm{MoO}_{3}$ nanoblades, respectively, which showed higher conductivity compared to previously reported values for similar morphologies. The obtained highly conductive $\mathrm{MoO}_{3}$ nanoblade film is favorable for high charge mobility in the next generation of electronics and in new generation batteries.

\section{Methods}

\section{Synthesis procedure and post-treatments}

Laterally grown $\alpha-\mathrm{MoO}_{3}$ nano-blades have been synthesized directly on seeded fluorine doped tin oxide substrates (FTO) (15 $\Omega \mathrm{cm}^{-2}$ ), via the hydrothermal technique. ${ }^{20}$ Briefly, $14 \times 14 \mathrm{~mm}$ FTO substrates were subjected to a standard cleaning procedure by sonication for $5 \mathrm{~min}$ in acetone and isopropanol each, followed by sonication in ethanol for $15 \mathrm{~min}$ and drying in a nitrogen flow. The cleaned substrates were treated with UV/ozone (Ossila) for $20 \mathrm{~min}$. The seed layer was deposited on the substrates consecutively from two evaporation sources: chrome plated tungsten rods (99.9\%) (Testbourne) and molybdenum(vi) oxide (99.98\%) powder (Sigma Aldrich) using thermal evaporation (Tectra mini-coater) at a base pressure of $<2 \times 10^{-6}$ mbar with an applied current and power of $15 \mathrm{~A}$ and $1 \mathrm{~kW}$, respectively. The thickness of the seed layer was $5 \mathrm{~nm} /$ $100 \mathrm{~nm}$ for $\mathrm{Cr} / \mathrm{MoO}_{3}$ and was monitored by a quartz microbalance with a resolution of $\pm 0.1 \mathrm{~nm}$. Finally, the seed layer coated substrates were annealed at $450{ }^{\circ} \mathrm{C}$ for $1 \mathrm{~h}$ in an ambient atmosphere. An aqueous solution of $\mathrm{Na}_{2} \mathrm{MoO}_{4} \cdot 2 \mathrm{H}_{2} \mathrm{O}(99.5 \%)$ (Sigma Aldrich) has been prepared in a PTFE autoclave liner to yield a total volume of $21.5 \mathrm{ml}$ and a concentration of $0.1 \mathrm{M}$. $0.29 \mathrm{~mol} \mathrm{~L}^{-1}$ hydrochloric acid $(\mathrm{HCl})(37 \%$ VWR chemicals) was added dropwise to the solution under continuous stirring for $10 \mathrm{~min}$. Then, the seed layer coated substrates were inserted upright into a PTFE holder in the precursor solution. Finally, the PTFE autoclave liner was sealed and put into a pre-heated oven at $180{ }^{\circ} \mathrm{C}$ for $210 \mathrm{~min}$. After the reaction, the autoclave was rapidly cooled down to ambient temperature in a water bath. The substrates of the vertically aligned $\alpha-\mathrm{MoO}_{3}$ nanoblades were washed with ethanol and allowed to dry under ambient conditions. The substrates are annealed separately for $3 \mathrm{~h}$ at $400{ }^{\circ} \mathrm{C}$ with a heating rate of $10{ }^{\circ} \mathrm{C} \mathrm{min}^{-1}$ in a digital controlled oven under different atmospheres, $\mathrm{O}_{2}, \mathrm{~N}_{2}$ and $\mathrm{H}_{2}(500 \mathrm{sccm})$ and under vacuum conditions.

\section{Characterization techniques}

Surface imaging was done using a Zeiss Cross Beam 1540XB field emission SEM using an acceleration voltage of $5 \mathrm{kV}$. X-Ray diffraction analysis XRD was performed using a Bruker AXS D8 Advance diffractometer employing a Bragg-Brentano geometry and $\mathrm{Cu}-\mathrm{K}_{\alpha}$ radiation. High resolution transmission electron microscopy (HR-TEM) images were obtained on a JEOL-2010 microscope operated at $200 \mathrm{kV}$. The TEM samples were prepared on holey carbon films Multi A TEM grids supplied by Quantifoil GmbH. Zero-loss filtered bright-field images and selected area electron diffraction (SAED) patterns were recorded. Using electron paramagnetic spectroscopy (EPR), spectra were recorded as follows: powder $\alpha-\mathrm{MoO}_{3}$ nanoblades were prepared using sonication of the vertically aligned grown $\alpha-\mathrm{MoO}_{3}$ nanoblades on the substrates in DI water for $3 \mathrm{~min}$. The powder was purified using centrifugation under $9000 \mathrm{rmp}$ for $20 \mathrm{~min}$. The obtained powder was divided to be annealed under different atmospheric conditions separately, as mentioned before for the substrates. Continuous-wave (cw) EPR spectra were recorded at the X-band $(\sim 9.3 \mathrm{GHz})$ on an Elexsys E580 EPR spectrometer (Bruker Biospin) equipped with a splitring resonator ER 4118X-MS3 (Bruker Biospin). The measurements were performed at $80 \mathrm{~K}$, which was realized in a gas-flow cryostat CF935O combined with a temperature controller ITC503 (both from Oxford Instruments) and using helium as a cooling agent. Experimental settings were adjusted to avoid any saturation and over modulation of the spectral lines. 
Typical experimental settings were: modulation amplitude of $1 \mathrm{G}$, modulation frequency of $100 \mathrm{kHz}$, microwave power of $0.64 \mathrm{~mW}$, magnetic field range of $1000 \mathrm{G}$, time constant of $40.96 \mathrm{~ms}$, conversion time of $40.96 \mathrm{~ms}$, number of points per scan 4096, and number of scans 4. All samples were measured in $3 \mathrm{~mm} / 2 \mathrm{~mm}$ (o.d./i.d.) quartz tubes (Technical Glass Products, Inc.). As prepared and treated $\alpha-\mathrm{MoO}_{3}$ nanoblades were placed as a powder and pressed slightly with a plastic piston from the top to avoid any occasional movement of the powder particles in the magnetic field. The weight of the powder sample was determined as a difference in masses of the empty quartz tube and the filled ones. The height of all samples in the quartz tubes was approx. $1 \mathrm{~cm}$, which corresponds to the length of the sensitive part of the resonator. The standard $\mathrm{CuSO}_{4}$ sample was prepared from $\mathrm{CuSO}_{4} \cdot 5 \mathrm{H}_{2} \mathrm{O}$ as a $10 \mathrm{mM}$ solution in a $\mathrm{H}_{2} \mathrm{O} /$ glycerol $(8: 2 \mathrm{v} / \mathrm{v})$ mixture; $30 \mu \mathrm{L}$ of this solution was transferred into a quartz tube and shock frozen in liquid nitrogen prior to the measurement. Spectral simulations were performed with the EasySpin software package for Matlab. ${ }^{61}$ The X-ray photoelectron spectroscopy XPS measurements were performed in ultrahigh vacuum (UHV) using standard Omicron XPS system with a Mg X-ray source. The samples were exposed to air before inserting into the UHV system. CASA XPS software was used for the fitting of the data. C 1s was used as a reference for the calibration of the data. Photoelectron spectroscopy in air (PESA) measurements has been carried out on an AC-2 instrument (Riken Instruments). The analysis has been performed at an excitation light power of $2400 \mathrm{nW}$ and scanned from $4.6 \mathrm{eV}$ to $6.2 \mathrm{eV}$ with a step size of $0.05 \mathrm{eV}$. The measurements have been recorded for three different positions on the substrate and repeated three times for each position. Temperature-dependent current-voltage characteristics have been measured using a home-made DC chamber, with an embedded ceramic heat element (SiNi type CLV) connected to a digital temperature controller TR400 with a CX-Thermo OMRON E58-CIFQ2. The temperature is adjusted digitally through a Pt-100 thermocouple that is fastened to the top of the substrates and connected to the temperature controller. The samples are measured in the temperature range of 30$130{ }^{\circ} \mathrm{C}$ with steps of $10{ }^{\circ} \mathrm{C}$ in $30 \mathrm{~s}$ and stabilization time of 5 min. Purpose-built current-voltage contacting probes were made of $\mathrm{Ni}$ foil covered with an evaporated thin layer of $\mathrm{Cr}$ as adhesion layer followed by a $150 \mathrm{~nm}$ thick Au layer (to reduce the resistance between the probes and our top electrodes on the vertically aligned $\alpha-\mathrm{MoO}_{3}$ nanoblades). As an extreme precaution, a Ni foil was used to make sure that the vertically aligned nanoblades are not destroyed by the weight of the contacting probes. A Keithley 2400 source meter was connected to the contacting probes and the data acquisition was controlled by a Matlab program. Two Au top electrodes of $120 \mathrm{~nm}$ thickness were directly evaporated through a mask onto grown and treated vertically aligned $\alpha$-MoO3 nanoblades. Diffuse reflectance spectra were recorded by a Cary5000 (Agilent technologies) from 400 to $800 \mathrm{~nm}$ in a $150 \mathrm{~mm}$ integrating sphere. The samples were housed in the back of integrated sphere where the incident light illuminates the samples perpendicularly.

\section{Conflicts of interest}

There are no conflicts to declare.

\section{Acknowledgements}

The authors sincerely acknowledge the German Academic Exchange Service (DAAD) and the Egyptian Ministry of Higher Education (General Administration of Mission) for funding the reported work. The work was supported by the Deutsche Forschungsgemeinschaft (DFG) through the Collaborative Research Center SFB 1214 project Z1 (Particle Analysis Center). The authors are grateful to Mr Tobias Seewald (Physics department, University of Konstanz) for his professional and precise revision of the article.

\section{References}

1 S. Tokito, K. Noda and Y. Taga, J. Phys. D: Appl. Phys., 1996, 29, 2750-2753.

2 J. Meyer, K. Zilberberg, T. Riedl and A. Kahn, J. Appl. Phys., 2011, 110, 033710.

3 K. Kanai, K. Koizumi, S. Ouchi, Y. Tsukamoto, K. Sakanoue, Y. Ouchi and K. Seki, Org. Electron., 2010, 11, 188-194.

4 P. A. Spevack and N. S. Mcintyre, J. Phys. Chem., 1992, 96, 9029-9035.

5 M. T. Greiner, L. Chai, M. G. Helander, W.-M. Tang and Z.H. Lu, Adv. Funct. Mater., 2013, 23, 215-226.

6 W. Gordy and W. J. O. Thomas, J. Chem. Phys., 1956, 24, 439-444.

7 E. D. Hanson, L. Lajaunie, S. Hao, B. D. Myers, F. Shi, A. A. Murthy, C. Wolverton, R. Arenal and V. P. Dravid, Adv. Funct. Mater., 2017, 27, 1605380.

8 A. Borgschulte, O. Sambalova, R. Delmelle, S. Jenatsch, R. Hany and F. Nuesch, Sci. Rep., 2017, 7, 40761.

9 M. T. Greiner, L. Chai, M. G. Helander, W. M. Tang and Z. H. Lu, Adv. Funct. Mater., 2012, 22, 4557-4568.

10 T. He and J. Yao, J. Photochem. Photobiol., C, 2003, 4, 125-143.

11 N. Li, Y. Li, W. Li, S. Ji and P. Jin, J. Phys. Chem. C, 2016, 120, 3341-3349.

12 S. Subbarayudu, V. Madhavi and S. Uthanna, ISRN Condens. Matter Phys., 2013, 2013, 1-9.

13 P. R. Huang, Y. He, C. Cao and Z. H. Lu, Sci. Rep., 2014, 4, 7131.

14 K. Inzani, T. Grande, F. Vullum-Bruer and S. M. Selbach, J. Phys. Chem. C, 2016, 120, 8959-8968.

15 Y. Li, X. Chen, M. Zhang, Y. Zhu, W. Ren, Z. Mei, M. Gu and F. Pan, Catal. Sci. Technol., 2019, 9, 803-810.

16 R. Kashfi-Sadabad, S. Yazdani, T. D. Huan, Z. Cai and M. T. Pettes, J. Phys. Chem. C, 2018, 122, 18212-18222.

17 K. Inzani, M. Nematollahi, F. Vullum-Bruer, T. Grande, T. W. Reenaas and S. M. Selbach, Phys. Chem. Chem. Phys., 2017, 19, 9232-9245.

18 H. Ding, H. Lin, B. Sadigh, F. Zhou, V. Ozoliņš and M. Asta, J. Phys. Chem. C, 2014, 118, 15565-15572. 
19 Y. Gong, Y. Dong, B. Zhao, R. Yu, S. Hu and Z. A. Tan, J. Mater. Chem. A, 2020, 8, 978-1009.

20 S. Z. Noby, K. K. Wong, A. Ramadoss, S. Siroky, M. Hagner, K. Boldt and L. Schmidt-Mende, RSC Adv., 2020, 10, 24119-24126.

21 International Centre for Diffraction Dat (ICDD).

22 A. A. Ramadan, A. A. Abd El-Mongy, A. M. El-Shabiny, A. T. Mater, S. H. Mostafa, E. A. El-Sheheedy and H. M. Hashem, Cryst. Res. Technol., 2009, 44, 111-116.

23 D. J. Goyal, C. Agashe, M. G. Takwale and V. G. Bhide, J. Cryst. Grow., 1993, 130, 567-570.

24 Y. Guo and J. Robertson, Appl. Phys. Lett., 2014, 105.

25 K. H. R. Tokarz-Sobieraj, M. Witko, A. Blume, G. Mestl and R. Schlögl, Surf. Sci., 2001, 489, 107-125.

26 C. G. Granqvist, in Handbook of Inorganic Electrochromic Materials, ed. C. G. Granqvist, Elsevier Science B. V., Amsterdam, 1995 , DOI: 10.1016/B978-044489930-9/50010-6, pp. 175-192.

27 Journal, 2007.

28 M. Labanowska, Phys. Chem. Chem. Phys., 1999, 1, 5385-5392.

29 Comments Inorg. Chem., 1984, 3(5), 230-238, DOI: 10.1080/ 02603598408080073.

30 K. Dyrek and M. Labanowska, J. Chem. Soc., Faraday Trans., 1991, 87, 1003-1009.

31 A. Varlec, D. Arčon, S. D. Škapin and M. Remškar, Mater. Chem. Phys., 2016, 170, 154-161.

32 E. Serwicka, J. Solid State Chem., 1984, 51, 300-306.

33 K. C. Radha, R. V. Anavekar, J. L. Rao and R. P. S. Chakradhar, Appl. Magn. Reson., 2008, 35, 1-13.

34 X.-S. Jia, C.-C. Tang, X. Yan, G.-F. Yu, J.-T. Li, H.-D. Zhang, J.-J. Li, C.-Z. Gu and Y.-Z. Long, J. Nanomater., 2016, 2016, 9102828.

35 N. S. Kopachevska, A. K. Melnyk, I. V. Bacherikova, V. A. Zazhigalov and K. Wieczorek-Ciurowa, Himia, Fizika ta Tehnologia Poverhni, 2015, 6, 474-480.

36 H. Pick, Ann. Phys., 1938, 423, 365-376.

37 H. Pick, Ann. Phys., 1940, 37, 421-428.

38 D. A. R. D. W. Lynch, Phys. Rev., 1968, 174, 1050-1059.

39 S. S. Sunu, E. Prabhu, V. Jayaraman, K. I. Gnanasekar, T. K. Seshagiri and T. Gnanasekaran, Sens. Actuators, B, 2004, 101, 161-174.
40 E. S. E. Haber, Polyhedrton, 1986, 2, 107-109.

41 C. Clausen, G. Skands, C. Bertelsen and W. Svendsen, Micromachines, 2014, 6, 110-120.

42 M. J. Toohey, Sens. Actuators, B, 2005, 105, 232-250.

43 C. Julien, A. Khelfa, O. M. Hussain and G. A. Nazri, J. Cryst. Growth, 1995, 156, 235-244.

44 V. Nirupama, K. R. Gunasekhar, B. Sreedhar and S. Uthanna, Curr. Appl. Phys., 2010, 10, 272-278.

45 R. K. Gupta, K. Ghosh and P. K. Kahol, Phys. E, 2010, 42, 1509-1512.

46 H. M. Chenari, H. Sedghi, M. Talebian, M. M. Golzan and A. Hassanzadeh, J. Nanomater., 2011, 2011, 1-4.

47 F.-C. Chiu, Adv. Mater. Sci. Eng., 2014, 2014, 1-18.

48 R. Aigner, A. Pointner, T. Preindl, P. Parzer and M. Haller, Presented in part at the Proceedings of the 2020 CHI Conference on Human Factors in Computing Systems, 2020.

49 R. Nigon, T. M. Raeder and P. Muralt, J. Appl. Phys., 2017, 121, 204101.

50 J. Yang, X. Xiao, P. Chen, K. Zhu, K. Cheng, K. Ye, G. Wang, D. Cao and J. Yan, Nano Energy, 2019, 58, 455-465.

51 H. A. Tahini, X. Tan, S. N. Lou, J. Scott, R. Amal, Y. H. Ng and S. C. Smith, ACS Appl. Mater. Interfaces, 2016, 8, 10911-10917.

52 I. G. Austin and N. F. Mott, Adv. Phys., 1969, 18, 41-102.

53 R. A. S. Y. P. Yadava, Mater. Chem. Phys., 1987, 17, 259-272.

54 S. K. S. Vipin Kumar, T. P. Sharma and V. Singh, Opt. Mater., 1999, 12, 115-119.

55 N. Illyaskutty, S. Sreedhar, G. Sanal Kumar, H. Kohler, M. Schwotzer, C. Natzeck and V. P. M. Pillai, Nanoscale, 2014, 6, 13882-13894.

56 M. Nowak, B. Kauch and P. Szperlich, Rev. Sci. Instrum., 2009, 80, 046107.

57 S. K. Deb and J. A. Chopoorian, J. Appl. Phys., 1966, 37, 4818-4825.

58 M. R. Tubbs, Phys. Status Solidi A, 1974, 21, 253-260.

59 H. Pick, Ann. Phys., 1940, 429, 421-428.

60 S. Deb, Philos. Mag., 1973, 27, 801-822.

61 S. Stoll and A. Schweiger, J. Magn. Reson., 2006, 178, 42-55. 